\title{
Anaemia independently predicts joint erosion in RA
}

Anaemia is associated with significantly increased rates of joint erosion in patients with rheumatoid arthritis (RA), according to research now published in the Annals of the Rheumatic Diseases. The results from this prospective cohort study suggest that anaemia could be useful as an independent predictor of radiographic progression.

"We wanted to know whether anaemia had, beyond its known statistical associations with signs and symptoms of $\mathrm{RA}$, really something to do with disease severity as indicated by X-ray progression", explains lead author Burkhard Möller. "Furthermore, we questioned whether anaemia could still be relevant with all the available biologic agents."

To investigate, the researchers analysed data obtained from 2,681 Swiss patients with RA, who were assessed over a mean period of 2.2 years between 1996 and 2007. Joint erosion was evaluated from 9,731 sets of radiographs of the hands and forefeet using the Ratingen score (for which bone surface area is assessed in 38 joints), and anaemia was defined using the WHO criteria. "We calculated multilevel mixed-effects linear regression models for prospectively collected and centrally assessed X-ray data, and adjusted for different confounding variables such as DAS28 or cDAI for disease activity and RA therapy", adds Möller.
Initial analyses, adjusted for potential confounding factors, found that radiographic progression was significantly faster in anaemic than non-anaemic patients $(P<0.001)$, and a correlation between erosion rate and anaemia severity was observed. "It was remarkable to see anaemia-related acceleration in joint damage progression in different analyses, despite all possible statistical adjustments for clinically assessed disease activity and treatment", comments Möller. A subgroup analysis reproduced these results in patients undergoing anti-TNF therapy $(n=1,020)$, suggesting that anaemia can still occur and have clinical consequences in these patients despite the known beneficial effects of TNF inhibitors in resolving RA-associated anaemia. Nonetheless, the researchers did observe a decrease (from $>24 \%$ to $~ 15 \%$ ) in the prevalence of anaemia in their total study population between 2001 and 2007, following the introduction of biologic agents for RA.

Importantly, the association between anaemia and radiographic progression was observed even in patients without clinically measurable disease activity. Thus, Möller proposes that anaemia could be used "as a novel and so far widely ignored indicator of subclinical disease activity". MRI or ultrasound imaging techniques should now be considered for disease activity

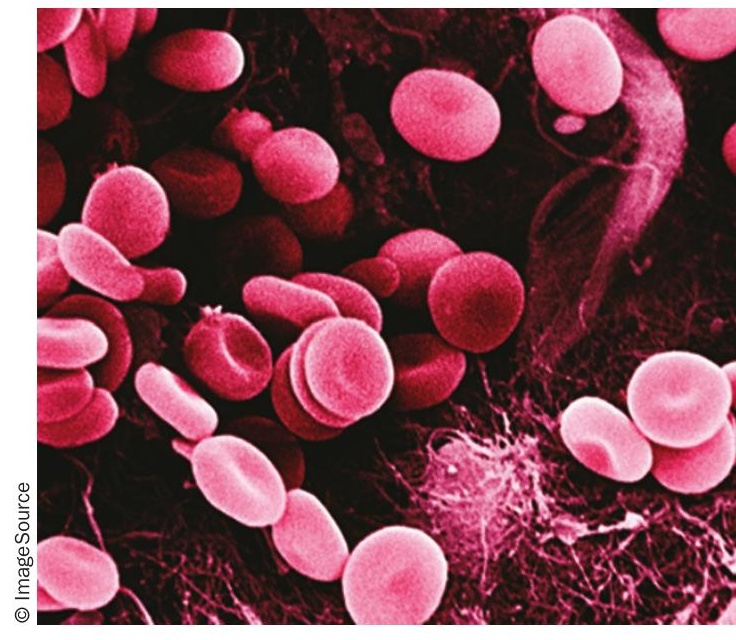

assessment in patients with anaemia that cannot be explained by iron deficiency or other RA-independent factors, he suggests. Fundamental experiments are still required to unravel the causality of anaemia, disease activity and joint damage in RA.

\section{Isabel Woodman}

Original article Möller, B. et al. Anaemia may add information to standardised disease activity assessment to predict radiographic damage in rheumatoid arthritis: a prospective cohort study. Ann. Rheum. Dis. doi:10.1136/ annrheumdis-2012-202709

Further reading Weiss, G. \& Schett, G. Anaemia in inflammatory rheumatic diseases. Nat. Rev. Rheumatol. 9 , 205-215 (2013) 